Basic Health Sciences

Poster

Abstract ID: 80

\title{
Co-expression of MYC and BCL2 in diffuse large B-cell lymphoma
}

\author{
Naznin Muhammad ${ }^{\mathrm{a}}$ | Ahmad Toha Samsudin ${ }^{\mathrm{b}}$ | Norlelawati A.Talib ${ }^{\mathrm{a}}$ | Aung Gyi ${ }^{\mathrm{a}}$ | Norra Harun ${ }^{\mathrm{c}}$ | \\ Suhaila Abdullah ${ }^{c}$ \\ ${ }^{a}$ Kulliyyah of Medicine, International Islamic University Malaysia

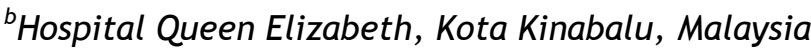

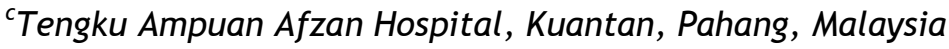

Introduction: Diffuse large B-cell lymphoma (DLBCL) is the most common type of nonHodgkin lymphoma. The pathogenesis of DLBCL is complex because it involves at least two different pathways, a de novo pathway and a transformation pathway. MYC and BCL2 oncogenes are 2 key regulators implicated in the pathogenesis. DLBCL with concurrent expression of MYC and BCL2 has been shown to be clinically aggressive and confers a worse prognosis. MYC detection by immunohistochemistry is however not performed in a routine diagnostic work up of DLBCL cases. This study examined the presence of MYC and BCL2 proteins by immunohistochemistry in patients diagnosed to have DLBCL. Methods: This retrospective study involved patients diagnosed to have DLBCL at Tengku Ampuan Afzan Hospital, Kuantan, Pahang (Year 2009-2011) and Queen Elizabeth Hospital, Kota Kinabalu, Sabah Malaysia (Year 2012-2014). Immunohistochemistry for MCY and BCL2 were performed on sections of formalin fixed paraffin embedded tissue blocks. Results: There were 91 cases analyzed. Forty-nine cases (53.8\%) exhibited concurrent expression of MYC and BCL2 proteins. In about one third of the cases, positivity was confined to BCL2. In 4 cases $(4.4 \%)$ only MYC was expressed while in 9 cases $(9.9 \%)$ both markers were negative. Overall about $60 \%$ and $85 \%$ of the cases were positive for MYC and BCL2 respectively. Conclusions: Approximately half of DLBCL case studied co-express MYC and BCL2. Prospective studies to look at the clinical significance and prognostic impact of this finding are advocated.

KEYWORDS: Diffuse large B-cell lymphoma, BCL2, MYC 